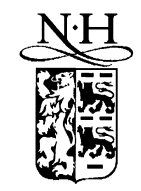

ELSEVIER

\title{
Pulse processing architectures
}

\author{
J. Basílio Simões*, Carlos M.B.A. Correia \\ Instrumentation Center of the Physics Department, University of Coimbra, P-3020 Coimbra, Portugal
}

\begin{abstract}
This paper introduces and discusses the features of a new multiprocessor architecture that aims at performing digital pulse processing at high throughput rates. It is based on a master Digital Signal Processor (DSP), special purpose trigger pulse locator and data routing circuits and an expandable array of slave peripheral DSPs. An historical perspective about the evolution of pulse spectrometry systems over the last 40 years is given. It is recognized that, up to the very recent commercial outcome of digital pulse processing systems, a tremendous evolution of technology but little change in their basic architecture has occurred. (C) 1999 Elsevier Science B.V. All rights reserved.
\end{abstract}

Keywords: Digital pulse processing; Pulse locator; Pulse height; Spectrometry

\section{Introduction}

Since the early 1950s pulse spectrometry has been playing around an important role in a number of scientific, industrial and medical situations. Pulse Spectrometry (PS) is particularly familiar to the experimental nuclear physicist since he generally deals with signal sources of a pulsed nature whose properties, namely the statistical ones, he wants to find out.

PS instrumentation has evolved ever since as the means to measure those properties as accurately as possible. Speed of operation also represents an important issue since it is directly related to the time needed to collect enough information to build up meaningful (or statistically accurate) spectra.

\footnotetext{
*Corresponding author. E-mail: jbasilio@gemini.ci.uc.pt.
}

The traditional function of a PS system consists of converting the electrical-charge pulses from the detector into voltage pulses and obtaining their amplitude distribution function. A dedicated instrument, the MultiChannel Analyzer (MCA), accomplishes this last task by dividing the amplitude space (generally associated to energy) in a number of equally spaced intervals, usually referred to as channels. Each channel holds the number of occurrences of the corresponding range of amplitudes. Each measured pulse amplitude leads to a one unit increment of the channel to which it is attributed. The number of the channels the system is capable to differentiate represents its energy resolution.

The earlier instruments did the job in a literal way using electromechanical means. This was the case of the so-called kick sorter of the 1950s that turns the pulse amplitude into a mechanical impulse that would eject a ball in an amplitude 
proportional way. The histogram was naturally built-up by the accumulation of balls in selfevident bins or channels (Fig. 1).

One funny consideration concerning this appliance is the physical coincidence between analog to digital conversion (considered as the ability to distribute a ball by a finite and discrete number of channels) and accumulation.

The advent of the electronic Analog-to-Digital Converter (ADC) removed the moving parts from MCAs and produced the huge valve units of the early 1960s that could histogram along 200 channels and store the spectra in its ferrite memory. By the end of this decade, however, MCAs started to incorporate a number of solid state devices and in the 1970s integrated circuits started to shrink the size of the MCA to the bench top level. The 1980s brought the microprocessor and by the end of this decade single board units, to be hosted by the ubiquitous PC, became available from a few manufacturers.

Looking back through this 30 -year period we can easily recognize the tremendous evolution of technology but little change in the basic architecture of the spectrometry systems. In fact, as it will be emphasized in Section 2, the same block diagram can describe the classical instrument along all that time.

The outcome, in the early 1990s, of fast precision ADCs combined with the high levels of integration of digital circuits has made it possible to devise a new approach to the design of PS systems. In the new design, focused in Section 3, the conventional analog front end is replaced by a digital pulse processor that computes pulse-height out from the data sampled by a fast high-resolution ADC.

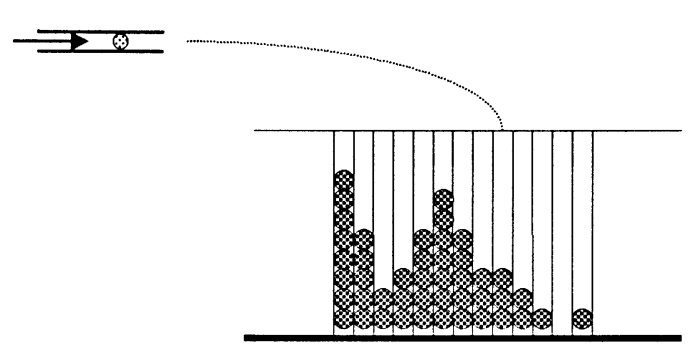

Fig. 1. The kick sorter at work.
Besides avoiding the typical non-linearities and instabilities of the analog front end, the digital approach holds an unsurpassed flexibility, inherent to the programmable nature of its performance. Under this new design it is possible to synthesize the theoretical optimum weighting function, taking into account the noise that is effectively present in the experimental set-up, and thus obtain the best energy resolution [1-3]. Moreover, effects such as pile-up [4], ballistic deficit [5] and charge trapping [6] can be corrected or eliminated at the processing level.

Although the most versatile and straightforward way to implement the digital pulse processor is through a software routine, the large computation time required by this approach has always limited its practical use. Then, to comply with the desired high throughput rates, some hardware digital pulse processor circuits have been recently developed [7-11]. This solution, in spite of sacrificing some versatility and resolution when compared with the software implementation, represents a clear advance over the traditional analog systems.

However, this is necessarily a transitory situation since the development of technology is continuously making available more powerful Digital Signal Processors (DSPs) running at increasingly higher clock frequencies. Based on the current expectations we can guess with great confidence that in the next decade a software-based digital pulse processing spectrometer will be capable of higher throughput rates than the current state-of-art analog Pulse-Height Analyzers (PHAs).

While such a powerful DSP is not available other solutions must be tried as the multiprocessor architecture presented and discussed in Section 4.

\section{Classical spectrometry system}

The classical analog spectrometer is depicted in Fig. 2. It includes the preamplifier, close to the detector, the linear shaping amplifier and the MCA. The MCA is mainly composed of the central peak stretcher with its companion linear gate, baseline restorer, current sources for discharging the store capacitor, and the ADC and memory to convert pulse-height to the digital domain and perform the 


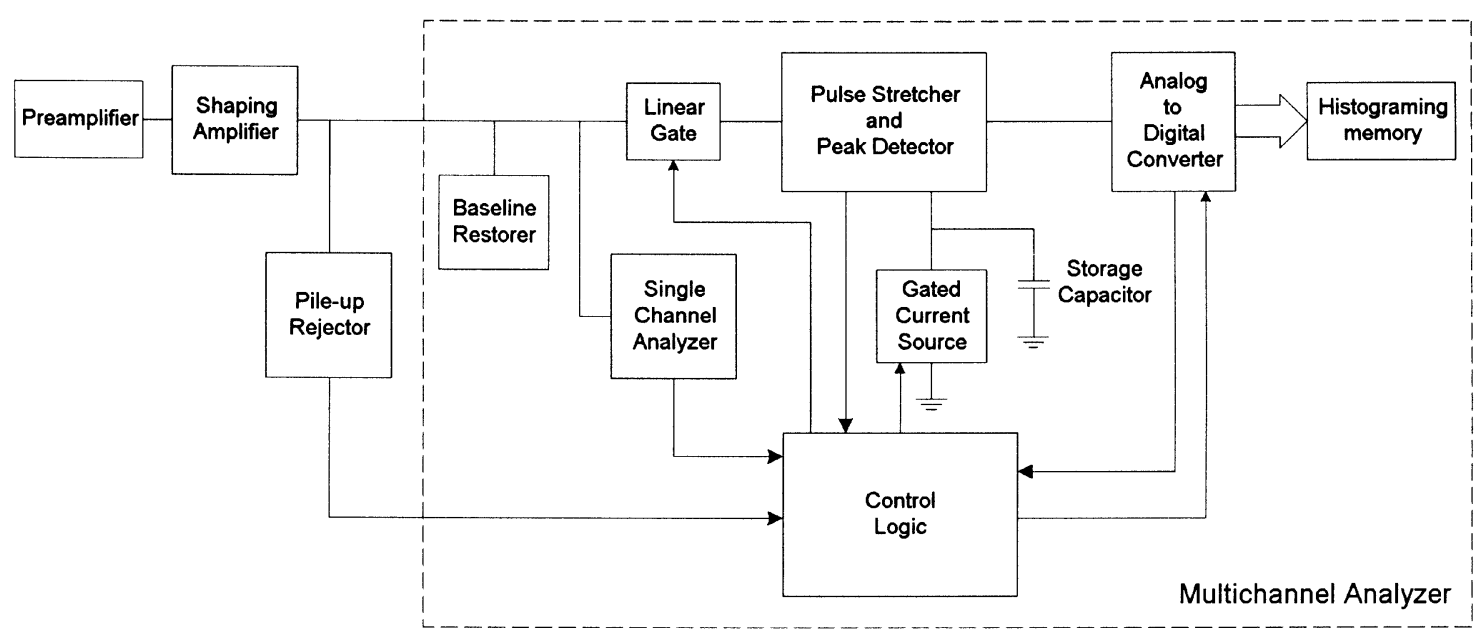

Fig. 2. Block diagram of the classical spectrometer.

histogram. Other building blocks could be optionally found: discriminators, pileup rejectors, peak stabilizers, etc.

The performance characteristics of MCAs, number of channels, linearity and dead time, are normally dependent on the specifications of the ADC. Thus, the technological evolution undertaken by MCAs has naturally followed that of ADCs. The first MCAs were based on Wilkinson-type ADC. Owing to their excellent linearity they are still used nowadays in systems with 4 or $8 \mathrm{k}$ channels using clock frequencies up to $300 \mathrm{MHz}$. However, conversion time is the main drawback of this kind of ADC which leads to long dead time MCAs.

The successive approximation type of ADC is much faster but has poor linearity. Its popularity in MCA applications has only been possible after the practical implementation of the sliding scale method [12] to improve its linearity $[13,14]$.

Flash-type ADCs provide a yet faster analog-todigital conversion but, at the current levels of integration, its number of channels and linearity are still incompatible with its application in the conventional MCA architecture. However, it was the outcome of flash ADCs with conversion times of a few tens of nanoseconds that finally triggered architectural changes in pulse spectrometry systems allowing its evolution towards the digital domain.

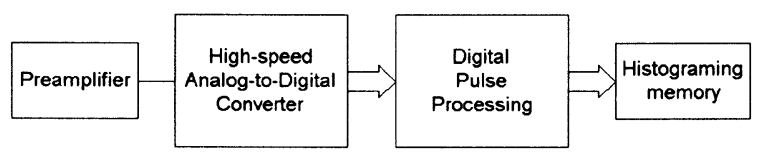

Fig. 3. Block diagram of the digital pulse processing spectrometer.

\section{Digital pulse processing}

The new digital pulse processing architecture (Fig. 3) is much simpler than the analog one. Pulses are sampled immediately after the preamplifier stage and are digitally processed afterwards. The advantages of this approach are well known for long time [15] and have been introduced with the appropriate references in Section 1.

As mentioned, this digital architecture allows the application of the optimum weighting function (WF) to the sampled pulse in order to get the best energy resolution under the experimental noise conditions. However, the large computation time demanded by the software implementation of the complete digital pulse processing chain led to the development of alternative solutions like:

(i) hardware implementation of the digital pulse processor [7-11]. This solution handles very high throughput rates but does not have enough flexibility to implement the theoretical optimum WF 
(which depends on the noise that is effectively present on the experimental set-up) and is also limited in what concerns pulse-shape analysis and correction of pulse distortions;

(ii) mixed solutions in which an analog pre-processor stage is used to shape and time limit the pulse before the sampling and digital processing stages $[16,17]$. This approach uses a DSP to run the desired pulse-processing routines, allowing for pulse rise-time discrimination [18] and other pulse shape analysis and correcting algorithms to be easily implemented. The analog pre-processor stage reduces the pulse duration and, hence, the number of samples needed to its digital representation and the corresponding processing time. This enables medium throughput rates to be achieved but does not completely avoid the disadvantages of using analog circuits.

In the next section we present and discuss the performance of a multiprocessor expansible architecture developed with the goal of performing alldigital pulse processing at high throughput rates.

\section{Multiprocessor digital pulse processing system}

The developed multiprocessor pulse spectrometry architecture (Fig. 4) is based on a master unit that connects to an expansible number of peripheral Slave Units (SU) through the communications, pulse transfer and control buses. The master unit contains, in addition to the DSP, a Trigger and Pulse Locator Unit (TPLU), a Bus Control Unit (BCU) and a very simplified analog front end.

The master DSP supervises the digitized pulse data transfers to the SUs, collects the processed pulse parameters from them and builds the energy spectrum. It also accommodates the user interface routines and is responsible for the download of the program code to the slave DSPs.

The analog front end is reduced to a linear amplifier state, a trigger unit and a fast and high-resolution digitizing block. Its digital output is temporarily stored in First-In-First-Out (FIFO) memories that function as the first of the several levels of pulse buffering implemented in this architecture.

The input signal is continuously digitized and transferred, through the pulse transfer bus, to the local FIFO of the selected SU. Each SU is identified by a different address located in the Slave Control Unit (SCU). When the FIFO of the selected SU gets full, a SU_busy signal is promptly issued by the SCU. As an immediate consequence, the BCU selects, through the control bus, the next

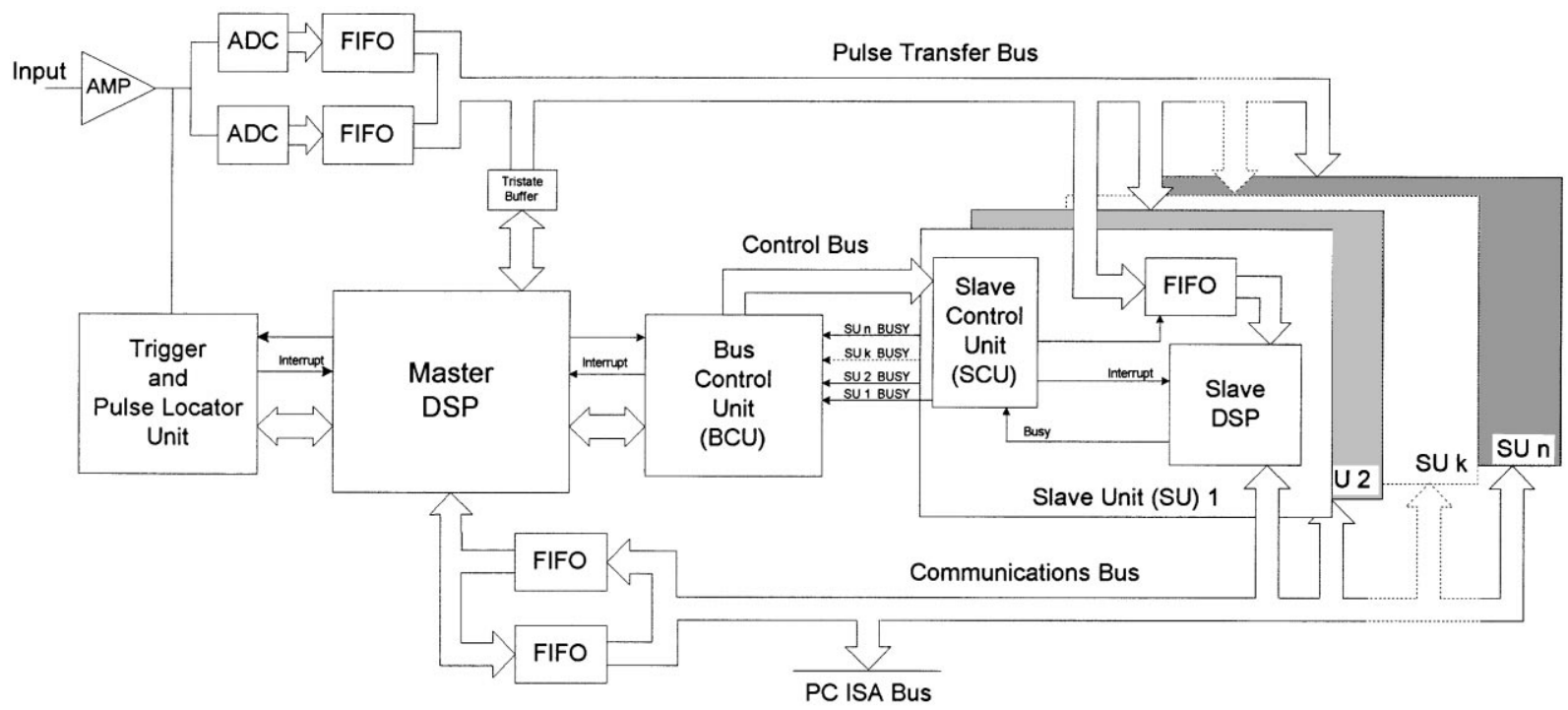

Fig. 4. Architecture of the multiprocessor pulse processing system. 
free SU and interrupts the master DSP signalizing the occurrence.

If the number of SUs is large enough to assure that at least one of them is always available to receive the digitized input signal, a virtual zero dead time spectrometry system can be obtained.

In the current implementation up to eight primary slaves are supported. However, a special router SCU was conceived to replace any of the SUs increasing the number of slaves in an unlimited tree fashion scheme. This router SCU is much similar to the master unit. It contains a DSP, a BCU, pulse transfer and communication buses and the companion FIFOs, and behaves like a SU to the master and like a master unit to their own (secondary) slaves.

The information concerning the arrival time of each pulse, furnished by the TPLU to the master, is stored in a lookup table associated with the selected slave [19]. This information will be passed to the correspondent $\mathrm{SU}$, through the communication bus, the next time it will be selected. Simultaneously, the SU sends to the master the available pulse processing results. These data exchanges do not introduce any dead time in the system because they are made in the time frame used to fill the FIFO of the SU with a new digital data stream coming from the pulse transfer bus.

All the slave DSPs are running the same pulse processing algorithm. The above-mentioned lookup table containing pulse's arrival times is used to program the Direct Memory Access (DMA) unit of the DSP to automatically transfer digital data relative to each pulse into the next available position on a circular queue that is defined in the DSP's memory [19].

The presented multiprocessor spectrometer is currently implemented in two PC boards using the low-cost floating point Texas Instruments TMS320C31 DSPs and an 80 MSPS, 12-bit, interleaved digitizing block with two Burr-Brown ADS800 ADCs.

The system is also prepared to be operated as a stand-alone spectrometer, that boot-loads its initializing program from an EPROM and uses the Ethernet to communicate with the PC.

A dedicated real-time operating system has been designed to this multiprocessor spectrometer. To accomplish the need for continuous and uninterrupted operation as well as the bounded response time constraints characteristics of real-time systems, the operating system was designed to comply with Posix.4 (extension of the Posix standard [20] for real-time systems).

Some of the programming techniques that have been employed are: use of coroutines to take care of all the processes that are simultaneously running; use of interrupts to handle the more time critical tasks; use of buffering data; use of a periodic timing interrupt routine and a scheme of semaphores associated with the main central task dispatcher routine to prevent deadlocks and properly reinitiate the system if necessary.

\section{Conclusions}

It was given an historical perspective about the evolution of pulse spectrometry systems over the last 40 years. Focusing on the recent developed digital pulse processing architectures, it was recognized the current difficulties to fully explore their advantages at reasonable throughput rates. Some compromise solutions have been analyzed and a new expansible multiprocessor architecture was presented. This new spectrometry architecture uses DSPs to perform all-digital pulse processing by running the appropriate software routines. The use of several levels of pulse buffering along with an expansible array of DSP-based slave units confers to this nuclear pulse processing system a very high throughput and an extremely reduced dead time.

\section{References}

[1] E. Gatti, M. Sampietro, P.F. Manfredi, Nucl. Instr. and Meth. A 287 (1990) 513.

[2] G. Bertuccio, A. Fazzi, A. Geraci, M. Sampietro, Nucl. Instr. and Meth. A 353 (1994) 257.

[3] A. Geraci, G. Ripamonti, A. Pullia, Conf. Record of the 1996 IEEE Nucl. Sci. Symp. Medical Imaging Conf. 1 (1997) 478.

[4] R.E. Chrien, R.J. Sutter, Nucl. Instr. and Meth. A 249 (1986) 421.

[5] A. Georgiev, W. Gast, IEEE Trans. Nucl. Sci. NS-40 (1993) 770 . 
[6] R. Hess, P. De Antonis, E.J. Morton, W.B. Gilboy, Nucl. Instr. and Meth. A 353 (1994) 76.

[7] F. Hilsenrath, H.D. Voss, J.C. Bakke, IEEE Trans. Nucl. Sci. NS-32 (1985) 145.

[8] T. Lakatos, Nucl. Instr. and Meth. B 47 (1990) 307.

[9] V.T. Jordanov, G.F. Knoll, A.C. Hubber, J.A. Pantazis, Nucl. Instr. and Meth. A 353 (1994) 261.

[10] M. Bordessoule, R. Bosshard, Nucl. Instr. and Meth. A 356 (1995) 452.

[11] EG\&G Ortec, DSPEC Digital Gamma-Ray Spectrometer, EG\&G 97/98 Modular Pulse-Processing Electronics and Semiconductor Radiation Detectors Catalog.

[12] C. Cottini, E. Gatti, S. Svelto, Nucl. Instr. and Meth. 24 (1963) 241.

[13] C.M.B.A. Correia, C.A.N. Conde, Nucl. Instr. and Meth. A 235 (1985) 536.
[14] C.M.B.A. Correia, J.C. Martins, Nucl. Instr. and Meth. A 290 (1990) 445.

[15] H. Koeman, Nucl. Instr. and Meth. 123 (1975) 161.

[16] J.B. Simões, P.C.P.S. Simões, C.M.B.A. Correia, IEEE Trans. Nucl. Sci. NS-42 (1995) 700.

[17] A. Geraci, G. Ripamonti, A. Pullia, Conf. Record of the 1996 IEEE Nucl. Sci. Symp. Medical Imaging Conf. 1 (1997) 478.

[18] P.C.P.S. Simões, J.F.C.A. Veloso, J.M.F. dos Santos, C.A.N. Conde, IEEE Trans. Nucl. Sci. NS-44 (1997) 521.

[19] J.B. Simões, J. Landeck, J.M.R. Cardoso, C.F.M. Loureiro, J.L. Malaquias, C.M.B.A. Correia, Conf. Record of the 1996 IEEE Nucl. Sci. Symp. 1 (1997) 448.

[20] IEEE Standard 1003.1 - 1990, IEEE Portable Operating System Interface for Computer Environments, IEEE, New York, 1900. 